\title{
Reversible Blindness Associated with Preeclampsia - A Report of Three Cases
}

\author{
Kathpalia $\mathrm{SK}^{1^{*}}$, Arora $\mathrm{CD}^{2}$ and Jain $\mathrm{AA}^{3}$ \\ ${ }^{1}$ Professor and HOD (Obstetrics and Gynecology), Andaman Nicobar Islands Institute of Medical Sciences, Port Blair, India \\ ${ }^{2}$ Professor (obstetrics and Gynaecology), Army College of Medical Sciences New Delhi, India \\ ${ }^{3}$ Resident (obstetrics and Gynaecology), Army College of Medical Sciences New Delhi, India
}

*Corresponding author: Kathpalia SK, Professor and HOD (Obstetrics and Gynecology), Andaman Nicobar Islands Institute of Medical Sciences, Port Blair, India, E-mail: kathpaliasukesh@gmail.com

Citation: Kathpalia SK, Arora CD, Jain AA (2018) Reversible Blindness Associated with Preeclampsia - A Report of Three Cases. J Nurs Healthcare Manag 1: 103

Article history: Received: 08 February 2017, Accepted: 07 May 2018, Published: 09 May 2018

\begin{abstract}
A large number of pregnancies are affected by hypertensive disorders of pregnancy. Preeclampsia is a systemic disease and affects almost all organs of the body. When visual cortex is affected the patient may develop blindness which is temporary and resolves within variable period of time. There is no need for any specific therapy for blindness; the standard management of severe preeclampsia is to be continued. Neuroimaging has a very limited role. Three cases that developed blindness in preeclampsia are presented.
\end{abstract}

Keywords: Blindness; Preeclampsia; Cortical

\section{Introduction}

Hypertensive disorders continue to be a major cause of maternal and perinatal mortality and morbidity1 especially in developing countries [1]. In India; 7 to $10 \%$ of all pregnancies are complicated by some kind of hypertensive disorders [2]. Preeclampsia is a syndrome peculiar to pregnancy, diagnosed when hypertension and proteinuria occur concurrently after twenty weeks of gestation [3]. It is a systemic disease and affects almost all maternal systems of the body like hematological, cardiovascular, renal, gastrointestinal and hepatic. It can affect neurological and cerebral systems also; which is responsible for convulsions in eclampsia. Visual symptoms like photophobia, visual spots, diplopia, and scotomata are suggestive of severity of the disease. Blurring of vision is the commonest visual symptom [4]. Eclampsia and preeclampsia can affect the visual pathways from the anterior segment to visual cortex [5].

Complete blindness is an occasional occurrence, affects 1 to $3 \%$ of eclampsia cases [6]. Blindness can occur due to the disease process affecting the occipital cortex retina or optic nerve [7-9]. Three cases of blindness in severe preeclampsia managed over two years in two different hospitals are presented.

\section{Case 1}

30 years old second gravida at 31 weeks and 3 days of gestation with one previous normal delivery; an unbooked case was brought to the hospital with vomiting, headache and blurred vision of one day duration. On general examination she was fully conscious and oriented, her BP was 192/120 mm of $\mathrm{Hg}$, had bilateral pedal edema of grade 2, systemic examination was normal. Ophthalmological examination showed no visual acuity, intraocular pressure not raised and fundoscopy did not show any abnormality. Obstetrical examination showed uterine height of 30 weeks with vertex presentation and normal fetal heart sounds. She was started on IV labetalol and oral methyl Dopa. Surfactant induction with steroids was initiated. Her BP got stabilized after about eight hours. Lab investigations were normal except proteinuria (2+ by dip stick) and low platelets $(90,000$ per ml). Next day she complained of abdominal pain with tenderness without any bleeding. Abruptio placenta was suspected though bedside ultrasound did not show any clear cut retro placental clot. In view of severe preeclampsia and suspicion of abruptio; she was taken up for emergency Cesarean Section (CS) under magnesium sulfate cover. CS was performed under general anesthesia. $1.4 \mathrm{Kg}$ baby was delivered and shifted to Neonatal Intensive Care Unit (NICU). Next day she complained of total blindness; case was reviewed along with eye specialist and neurophysician and diagnosis of cortical blindness was made. CT scan showed hypodense areas in occipital 
cortex (Figure 1). MRI done next day showed findings suggestive of vasogenic edema involving the white matter of both parieto occipital lobes (Figure 2). Imaging studies were suggestive of PRES (posterior reversible encephalopathy syndrome). She regained her vision two days later. During intra and immediate postoperative period her BP varied between 150-160/100-110mm of Hg. Her BP became normal after ten days and methyl Dopa was stopped. She was discharged after five weeks when baby became fit for discharge from hospital.

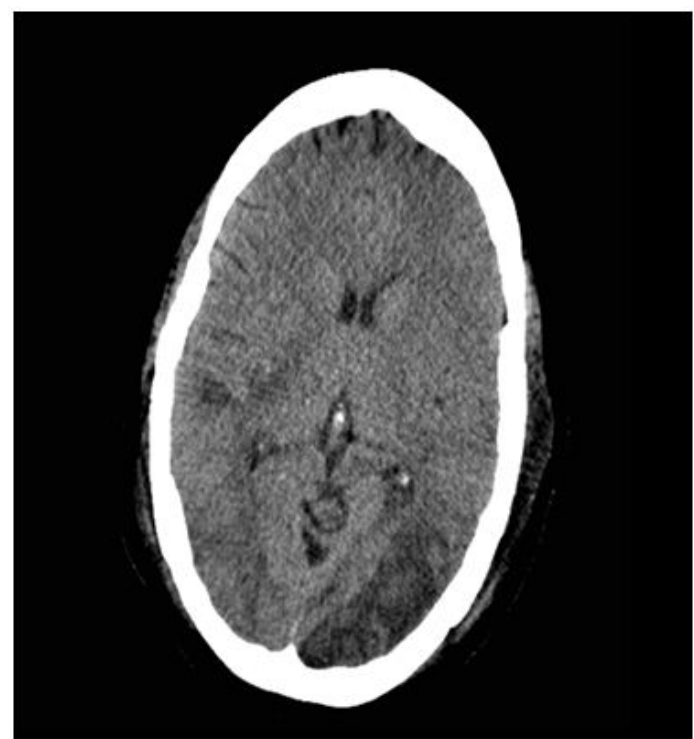

Figure 1: CT Scan showing hypodense areas in parieto occipital lobes
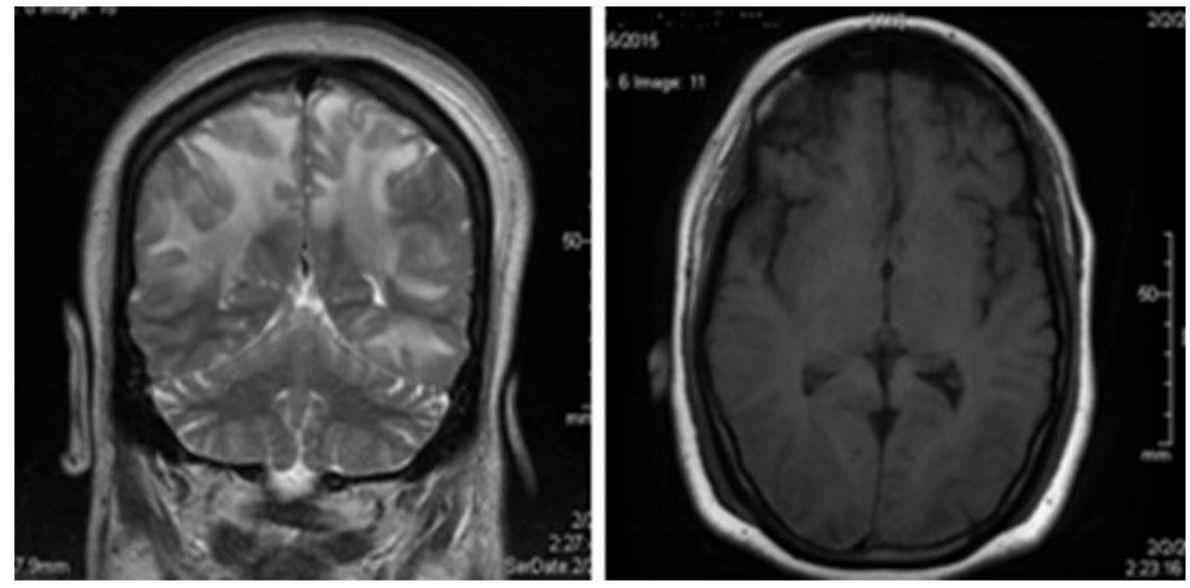

Figure 2: MRI showing edema involving the white matter

\section{Case 2}

32 years old, third gravida with previous one CS and one first trimester abortion was a booked case with irregular visits. At 34 weeks of gestation; she developed complete blindness after a convulsive fit at home in the morning and was brought to hospital within 30 to $40 \mathrm{~min}$ after the convulsive fit. When she was brought to the hospital she was drowsy and complained of blindness. Her BP was 160/106 mm of $\mathrm{Hg}$, minimal pedal edema was present and she was afebrile. General and systemic examination including neurological examination was normal. Detailed ophthalmological examination including pupillary reaction and fundoscopy were normal but visual acuity was absent. Obstetrical examination showed uterine height of 32 weeks, vertex presentation without scar tenderness; not in labour, fetal heart was heard normally. Cervix was unfavorable. Urine showed $3+$ proteinuria but all other investigations were normal. She was started on Magnesium sulfate (Pritchard regimen); and planned for CS under epidural anesthesia. She had another convulsion while on OT table; hence epidural anesthesia was abandoned and surgery performed under general anesthesia. A female baby weighing $1.7 \mathrm{~kg}$ was born with good Apgar score. Post operatively magnesium sulfate was continued for $24 \mathrm{~h}$. She recovered her vision the next day; that is within $30 \mathrm{~h}$, BP became normal on fourth day. Her post operative period was uneventful and was discharged after three weeks when baby became more than $2 \mathrm{~kg}$. Neuroimaging could not be done as it was not available.

\section{Case 3}

25 years old second gravida at 39 weeks with one previous normal delivery; an unbooked case was brought to hospital with headache and total blindness of two to three hours duration. She had vomited once at home. On examination she was conscious but drowsy; 
her BP was 180/130 mm of Hg. She had Grade 4 pedal edema, rest of general and systemic examination was normal. Neurological examination revealed brisk knee jerks. Ophthalmological examination showed normal pupillary reaction; normal intraocular pressure and normal fundoscopy but visual acuity was absent. Obstetrical examination was corresponding to normal findings at term and cervix was favorable. Urine showed 2+ proteinuria and platelet count was 110000 per ml; all other investigations being normal. She was started on magnesium sulfate therapy and her BP was brought down with IV labetalol. Since her cervix was favorable; she was induced with oxytocin drip and delivered a full term baby within six hours. Magnesium sulfate was continued postnatally for $24 \mathrm{~h}$ and oral antihypertensives were started. Her vision returned after $48 \mathrm{~h}$ and BP became normal after ten days and she was discharged to home. Repeat urine and blood examination were normal at that time. Neuroimaging was not done as her relatives could not afford.

\section{Discussion}

Preeclampsia/eclampsia is a systemic disease where each and every organ can be affected; including visual pathways. Once the visual pathway is involved the patient can have many visual disturbances; blindness being the most scary and severe. Earlier it was thought that blindness in preeclampsia occurs due to retinal involvement but now it is believed to be due to involvement of occipital region of cerebral cortex hence called cortical blindness [8]. Loss of vision due to cortical involvement is always bilateral while it may not be so in retinal involvement. Blindness may occur with or without convulsions, either before or after. It can occur before or after delivery as it happened in our series. Cortical blindness is characterized by presence of intact pupillary reflex and normal fundoscopy findings. Vision is usually regained within 4 hours to 8 days [10]. In our series also; all the cases had regained their vision during this period.

The basic etiology is vasospasm which increases resistance to blood flow; and accounts for development of hypertension. This vasospasm results in disparity in cerebral regional blood flow affecting the posterior circulation called PRES, this occurs due to failure in the autoregulation of posterior cerebral arterioles followed by extravasation of fluid into brain tissue [8]. Posterior cerebral vessels seem more susceptible to these changes due to less abundant sympathetic innervations.

Role of imaging: Grimes, et al., in 1980 reported first case in which computed tomographic scanning was used to demonstrate reversible cortical lesion in a woman with preeclampsia and cortical blindness [11]. Neuroimaging findings in cortical blindness range from normal to typical findings such as hypodensity on CT or hyperdensity on T2-weighted MRI [12]. Neuroimaging may be performed to pick up any other lesion and it is of immense prognostic value but is not mandatory too be carried out in every case. Management of preeclampsia with or without blindness remains the same; which includes Mag Sulphate therapy, control of $\mathrm{BP}$, care of an unconscious patient and termination of pregnancy as soon as possible.

Sudden and complete blindness is terrifying but fortunately it is reversible; therapy remains the same as for severe preeclampsia with or without blindness. The patient and relatives should be reassured about the good prognosis.

\section{References}

1. Steegers EA, von Dadelszen P, Duvekot JJ, Pijnenborg R (2010) Pre-eclampsia. Lancet 376: 631-44.

2. GB Doddamani, UG Doddamani (2014) Perinatal outcome in preeclampsia: a prospective study. Scholar J Appl Med Sci 2: 291-3.

3. B Sibai, G Dekker, Kupfermic M (2005) Pre-eclampsia. Lancet 365: 785-99.

4. Omoti AE, Waziri-Erameh JE, Okeigbemen VW (2008) A review of changes in the ophthalmic and visual system in pregnancy. African J Reprod Health 12: $185-96$.

5. Samra KA (2013) The eye and visual system in the preeclampsia/eclampsia syndrome. What to expect? Oman J Ophthalmol 6: 87-91.

6. Jyotsana, Sharma AK, Bhatt S (2004) Reversible blindness in severe preeclampsia. JK Science 6: 43-5.

7. Swende TZ, Abwa T (2009) Reversible blindness in fulminating preeclampsia. Ann Afr Med 8: 189-91.

8. Cunningham FG, Fernandez Co, Hernandez C (1995) Blindness associated with preeclampsia and eclampsia. Am J Obstet Gynecol 172: 1291-8.

9. S Tayade, A Wattamwar (2014) Bilateral detachment in pregnancy complicated by preeclampsia and placental abruption. Int J Biomed Res 5: 780-2.

10. Rajaram S, Goel N (2002) Sudden bilateral loss of vision postpartum. J Indian Acad Clini Med 3: 213-4.

11. Grimes DA, Eckbladh LE, McCartney WH (1980) Cortical blindness in preeclampsia. Int J Gynecol Obstet 17: 601-3.

12. Apollon KM, Robinson JN, Schwartz RB, Norwitz E (2000) Cortical blindness in severe preeclampsia: computed tomography, magnetic response imaging and single photon-emission computed tomography findings. Obstet Gynecol 95: 1017-9. 